\title{
OIL BODIES IN LIVERWORTS (HEPATICAE)
}

\section{S.R. GRADSTEIN}

Instituut voor systematische plantkunde, Utrecht

A characteristic feature of the liverwort cell is the presence of "oil bodies". Chemically they are made up of rather volatile terpenoids, mainly sesquiterpenes and their derivatives (SUIRE 1975) which seem to be surrounded by a membrane. The nature of this membrane is still unknown.

Through the light microscope the oil bodies appear as colourless, rarely brownish or blueish organelles in the cytoplasm, showing great variation in shape, size, number and structure. The morphological characteristics of the oil bodies, which have been studied over $80 \%$ of the genera of Hepaticae, have been shown to be taxonomically significant (cf. SCHUSTER 1966). While in the subclass Marchantiidae they are restricted to special, chloroplast-free "oil cells" of the gametophyte, in the remaining groups (subclass Jungermanniidae) they are abundant in the green cells of gametophyte and sporophyte. In the course of our investigations on tropical American liverworts we recognized four morphological types (GraDSTEIN et al. 1977):

1) Massula type - oil bodies homogeneous, small $(2-6 \mu \mathrm{m})$ and numerous, in median leaf cells more than 8 per cell.

2) Bazzania type - oil bodies homogeneous, large (4-15 $\mu \mathrm{m}$ long) and few, up to 8 per cell.

3) Jungermannia type - oil bodies finely segmented, consisting of numerous minute, indistinct globules surrounded by a common membrane. Size and number variable.

4) Calypogeia type-oil bodies coarsely segmented, consisting of distinct globules aggregated to form a "grape-cluster"; a common membrane seemingly lacking. Size and number variable.

Taxonomically, it appears that small genera as a rule have only one oil body type, which in about $2 / 3$ of the liverwort genera is the Jungermannia-type. Larger genera, e.g. Calypogeia, Lophozia, Plagiochila, may have two or even three different types, which serve to characterize sections or subgeneric divisions in correlation with other morphological characters. In practice this means that comparative studies on oil bodies help to arrive at better and more natural species arrangements; these studies should be particularly rewarding for work on taxonomically poorly investigated or "difficult" groups.

The volatile nature of the chemical components of the oil body, however, causes rapid disintegration of the organelle in dry or fixated material. We discovered that in the various oil body types the process of disintegration differs and may cause errors. For example, Massula-type oil bodies become vaguely and finely segmented upon degeneration, thus resembling the Jungermannia type, 
whereas the Jungermannia-type may resemble the Massula type before disintegration. Thus it appears that for taxonomic studies the use of fresh material is absolutely required.

As to the development of the oil bodies it has long been debated whether they originate from vacuoles or from certain cytoplasmatic organelles. Although a "cytoplasmatic origin" was more uniformly accepted to be correct, limiting techniques left the question unsettled for a long time. Recent EM-studies by Crandall-StOTLER (1971) showed that in Radula obconica oil bodies develop in the youngest cells through fusion of small osmiophilic droplets originating from chloroplasts. Her studies also suggest that the oil bodies function in the storage of reserve photosynthate, although arguments for a primarilyecological function, such as protection against frost, desiccation or insect attack have been put forward by previous authors.

The chemical constitution of the liverwort oil bodies, finally, is a subject of increasing interest among chemists. Although numerous papers on the terpenoid compounds of liverworts have appeared in the last decade (HuNECK 1977), only a very small portion of the known species and genera has been investigated. Most of the sesquiterpenoids identified thus far are novel and probably taxonomically relevant, such as the allergenic sesquiterpene-lacton frullanolide of many Frullania species, which may cause contact-dermatitis. Other compounds have been shown to be responsible for the specific taste or odour some liverwort species possess. It is evident that the chemotaxonomic significance of oil body constituents remains a fruitful subject for future investigations.

\section{REFERENCES}

CRANDALl-StOTLER, B. J. (1971): Light, ultrastructural, and chemical investigations of the oil-bodies of Radula obconica.Presented at the Canadian Botanical Association - American Institute of Biological Sciences joint meeting at the University of Alberta, Edmonton, Canada.

Gradstein, S. R., A. M. Cleef \& M. H. Fulford (1977): Studies on Colombian Cryptogams 11. Oil body structure and ecological distribution of selected species of tropical Andean Jungermanniales. Proc. Kon. Ned. Akad. Wetensch. (C) 80: 377-420

Huneck, S. (1977) Neue Ergebnisse zur Chemie der Moose, eine Übersicht 5. Journ. Hattori Bot. Lab. 43: 1-30.

Schuster, R. (1966): Oil bodies. In: The Hepaticae and Anthocerotae of North America I. New York.

SuIRE, C. (1975): Les données actuęlles sur la chimie des bryophytes. Rev. Bryol. Lichénol. 41 : $105-256$. 